\title{
Measurement of Spin Motions in a Storage Ring Outside the Stable Polarization Direction*
}

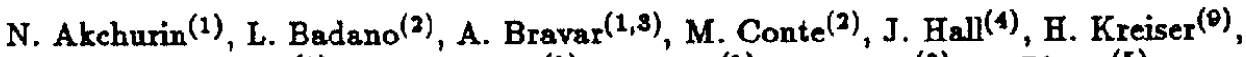 \\ J. MePherson ${ }^{(1)}$, F. Olchowski(1), Y. Onel (1), A. Penzo ${ }^{(3)}$, A. Pisent ${ }^{(5)}$, \\ M. Pusterla ${ }^{(8)}$, T. Rinckel ${ }^{(7)}$, R. Rossmanith ${ }^{(8)}$ \\ (1) Department of Physies, University of Iowa, Iowa City, IA 52242, USA \\ (2) Dipartimento di Fisica dell'Universita di Genova, INFN Sezione di Genova, \\ Vis Dodecaneso 33, I-16146 Genova, Italy \\ (3) INFN Sezione di Trieste, Via Valerio 2, I-34127 Trieste, Italy \\ (4) University of New Mexico, Albuquerque, New Mexico, USA \\ (5) INFN LNL, Via Roma 4, 35020 Legnaro (PD), Italy \\ (6) Dipartimento di Fisica dell'Universita di Padova, INFN Sezione die Padova \\ Via Marzolo 8, I-35131 Padova, Italy \\ (7) IUCF, 2401 Milo B. Sampson Lane, Bloomington, Indiana 47408, USA \\ (8) CEBAF, 12000 Jefferson Ave., Newport News, Virginia 23606, USA
}

(9) I. Inst. fuer Experimentalphysik, Luruper Chaussee 149, D-2000 Hamburg 52, Germany

\section{Abstract}

Polarized, stored beams are becoming a more and more important tool in nuclear and high energy physics. In order to measure the beam polarization in a storage ring the polarization vector of the stored beam has to aim, revolution for revolution, over a period of seconds to minutes, into the same, so-called "stable", direction. In this paper measurements at the Indiana University Cooler Ring (IUCF) are described in which for the first time in a storage ring oscillations of the polarization vector around this stable direction have been measured [1]. The existence and the dynamics of such oscillations are, for instance, important for a new proposed technique for polarizing stored hadron beams [2].

\section{INTRODUCTION}

The behaviour of polarized beams was studied both in electron-positron storage rings $[3,4]$ and proton storage rings [5].

The theoretical description of the polarization in these storage rings is mainly based on a formalism introduced by Derbenev and Kondratenko [6] and later by Chao [7]. The basic concept is the following: all the trajectories in a storage ring can be calculated relative to the closed orbit, a (fictive) trajectory, which repeats itself from one revolution to another. The behaviour of the polarization can be calculated in a similar way. A polarization vector is found along this closed orbit which also repests itself from one revolution to another. This vector is called $\mathbf{n}$-axis.

The thinking in terms of an ever-recurring polarization direction is highly supported by the polarimetry used in storage rings. In order to measure polarization with sufficient accuracy, the measurements have to be performed over several seconds or minutes [8].

\footnotetext{
"This work is supported in part by the U.S. Department of Energy, DOE-Grant No: DE-FG02-91ER10644, The University of Iowa Carver Scientific Research Grant and INFN, Italy
}

In this paper the first measurements on the stability of time-varying spin components are reported. The practical importance of an oscillating polarization is described somewhere else $[2,9]$. The measurements were performed at the Cooler Ring of the Indiana University Cyclotron Facility (IUCF) in Bloomington, Indiana. Recently a Siberian Snake was installed in this ring for demonstration purposes [5]. The snake consists of a solenoid which rotates the polarization by 180 degrees around the momentum axis and skew quadrupoles. The skew quadrupoles are located on each side of the solenoid. The authors of this paper used this Snake, the existing polarized source and the existing polarimeter $[10]$ to measure time-dependent spin motions.

\section{THE STABLE SPIN DIRECTION}

Particle and spin motion are related to each other. When the particle is deflected by an angle $\alpha$ around a certain axis its spin is rotated around this axis by an angle $\psi:$

$$
\frac{g-2}{2} \gamma \alpha=G \gamma \alpha=\psi
$$

where $g$ is the proton $g$ factor and $\gamma$ the Lorentz factor. The numerical value of $G$ is 1.7928 . Equation (1) is a direct consequence of the well known BMT-equation on the behaviour of the spin $\vec{S}$ in a magnetic field [11]:

$$
\frac{d \vec{S}}{d t}=\frac{e}{\gamma m} \vec{S} \times\left[(1+G \gamma) \vec{B}_{\perp}+(1+G) \vec{B}_{\|}\right]
$$

For the experiment an energy was chosen in which the spin performs two revolutions during one revolution of the beam: $G \gamma=2$. For a machine consisting only of bending magnets the energy corresponding to $G \gamma=2$ is $108.4 \mathrm{MeV}$. A more careful analysis $[5,12]$ showed that the solenoid of the cooler also contributes to the spin tune and the correct energy for $G \gamma=2$ is $106.2 \mathrm{MeV}$. The solenoid of the Siberian Snake rotates the spin around the momentum axis by an angle of 180 degrees. The required field strength for a 180 degree rotation can be derived from equation (2). 
The $n$-axis of a machine with a Siberian Snake can be derived from fig. 1 .

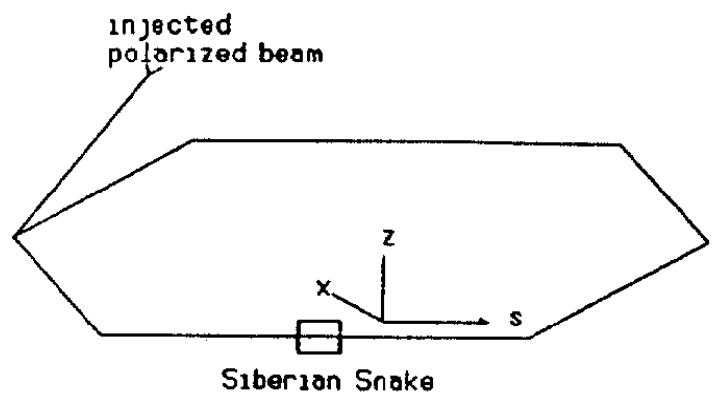

Fig. 1 The coordinate system. A spin manipulation system in the injector channel allows the injection of the beam in any polarization direction. For the experiment a polarization vector parallel to the vertical direction was chosen.

Using the coordinate system $[x, s, z]$ defined in this figure and choosing $\psi(s)=0$ at the snake, the $n$-axis is

$$
\vec{n}=(\sin (\psi(s)), \cos (\psi(s), 0)
$$

$s$ is the path length of the trajectory and $\psi(s)$ is the spin precession angle. The spin rotation only takes place in the bending magnets. The expression $\psi(s)$ describes the non-uniform spin advance in the machine.

\section{THE EXPERIMENT}

A beam polarized along the z-axis is injected into the storage ring. The beam is cooled by an electron beam and stored for circa 10 seconds. After this time, the beam is directed towards the target of the polarimeter [10]. The target is a $4.5 \mathrm{~mm}$ thick graphite slab. The transverse tail of the bunch is scattered. During the next revolutions the center of the beam is brought gradually closer to the target until the whole beam intercepts with the target (fig. 2). Afterwards a new beam is injected and the measurement is repeated.

According to equation (3) the $\mathrm{n}$-axis of the ring with the Snake is in the horizontal plane. The polarization $\vec{P}$ of the injected vertically polarized beam oscillates therefore around the n-axis:

$$
\vec{P}=P_{0}\left(0,0,(-1)^{m}\right)
$$

where $m$ is the number of revolutions. For a moment energy oscillations are neglected.

A standard polarimeter integrating over many revolutions would find that the beam is unpolarized: in the time average the vertical spin direction cancels. In order to measure this time varying polarization the polarimeter has to be gated in such a way that data are taken only every second revolution (fig. 2). The gate is opened every second revolution for half a revolution. If there is no depolarization, the polarimeter should measure a vertical polarizstion.

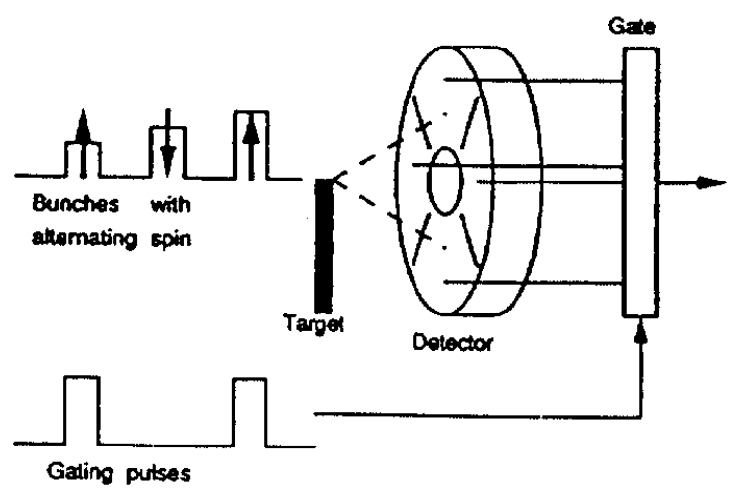

Fig. 2 The polarimeter and the gating system. The beam is directed to a carbon target and the asymmetry in the distribution of the scattered particles is measured [10]. Both the horizontal and the vertical polarization are measured at the same time. The polarimeter is gated in such a way that data are taken every second revolution.

Synchrotron oscillations are energy oscillations around the nominal energy.

$$
\left(\frac{\Delta \gamma}{\gamma}\right)=\left(\frac{\Delta \gamma}{\gamma}\right)_{0} \sin (\omega, t+\delta)
$$

where $\omega_{\text {s }}$ is the synchrotron frequency. Although the energy deviations are small these oscillations change the measurement in the following way:

The spin rotation in the solenoid for particles with energy deviation is no longer 180 degrees. Particles with higher (lower) energies then the nominal energy are rotated less (more) than 180 degrees according to equation (2).

This effect changes the results of the measurements significantly. Due to the energy oscillations the spin deviates from the vertical axis by

$$
u=\frac{1}{n} \sum_{n} \sin \left(2 \pi n+A \sin \left(2 \pi \omega_{0} t_{0}+\delta\right)\right)
$$

when the polarimeter only takes data every second revolution. $t_{0}$ is the revolution time and $A$ is a measure for the magnitude of the energy deviations. As a result the energy oscillations reduce the degree of the measurable polarization. The degree of reduction depends on the magnitudes of $A$ and $\omega_{3}$. The degree of the measurable polarization increases with $\omega_{0}$.

In the following the rotation angle of the solenoid is changed from 180 degrees to $180+\beta / 2$ degrees. For the same measurement as before, equation (6) has to be rewritten in the following way

$$
u(\beta)=\frac{1}{n} \sum_{n} \sin \left(2 \pi n+n \beta+A \sin \left(2 n \omega_{,} t_{0}+\delta\right)\right)
$$


$\beta$ is changed by changing the current through the solenoid. In fig. 3 the polarization $u(\beta)$ is plotted as a function of $\beta$ (=solenoid current). A current of $145.5 \mathrm{~A}$ corresponds to a spin rotation of 180 degrees. A lower current means less than 180 degrees rotation and a higher current more than 180 degrees rotation. It was assumed that the original polarization of the injected beam was $80 \%$ parallel to the vertical direction (as in the experiment). The distance between the center frequency and the two sidebands corresponds to a synchrotron tune of $Q,=0.004$ (at which the machine operates).

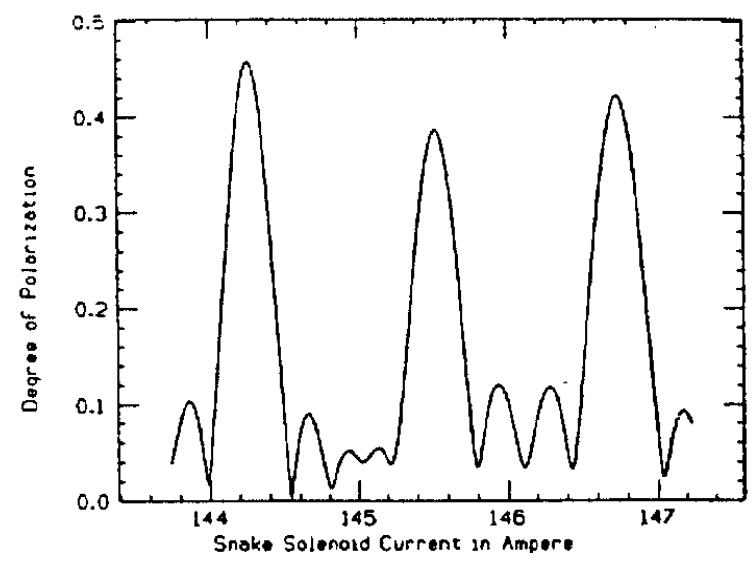

Fig. 3 Simulation of the measurement. The injected beam is $80 \%$ vertically polarized. The polarimeter measures the transverse component of the polarization. The polarimeter is gated in such a way that data are taken only every second revolution of the beam. The two peaks centered around the main peak are caused by synchrotron oscillations.

Fig. 4 shows the results of the measurements. The position of the side peaks relative to the main peat is in excellent agreement with the simple assumptions developed in equations (6) and (7).

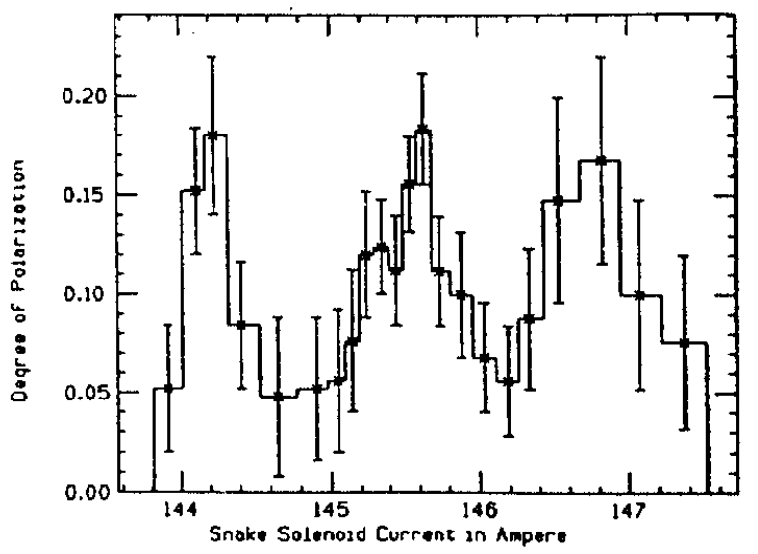

Fig. 4 The results of the measurements. The 3 peaks are separated by the amount predicted in fig. 3. The absolute height is smaller by a factor of 2 compared with the calculations. This deviation has to be investigated in more detail in the future.

In summary, it was demonstrated for the first time that oscillations of the polarization around the stable direction exist and can be measured.

The authors wish to thank Prof. Cameron from the Indiana University Cooler Ring in Bloomington for his hospitality. We would also like to thank Prof. A. Krisch and his team for the use of their equipment. Two of us (H. K. and R.R.) would also like to express their thanks to Prof. U. Strohbusch from the University of Hamburg for his support and help and finally one of us (R.R.) would like to thank Prof. Leemann of CEBAF, Virginis for support and encouragement.

\section{REFERENCES}

[1] N. Akchurin et al., Phys. Rev. Lett., 69,1753(1992).

[2] T. Nünikoski and R. Rossmanith, Nucl. Instrum. Methods A255,460(1987). Y. Onel, A. Penzo and R. Rossmanith, Proc. VII International Symposium on High Energy Spin Physics, Protvino 1986.

[3] R. Schmidt, AIP Proc. 252, 1991 Accelerator Instrumentation Workshop, Newport News, Virginia, 1991.

[4] A. A. Sokolov and I. M. Ternov, Sov, Phys, Dokl. 8,1203(1964).

[5] A. D. Krisch et al., Phys.Rev.Lett.63,1137(1989).

[6] Y. Derbenev and A. M. Kondratenko, Sov. Phys. JETP 37, 968(1973).

[7] A. Chso, Nucl. Instrum. Methods 180,29(1981).

[8] M. Placidi et al., Proc. EPAC Conf., Nice 1990.

[9] J. P. Koutchouk, CERN Internal Note SL/AP Note $16,1991$.

[10] B. von Przewoski et al., Proc. 9th Int. Symp. on High Energy Spin Physics, Bonn, Sept. 1990.

[11] V. Bargmann, L. Michel, and V. L. Telegdi, Phys. Rev. Lett. 2,435(1959).

[12] M. G. Minty, Studies of Siberian Snakes and Depolarizing Resonances at the Indiana University Cyclotron Facility Cooler Ring, Thesis, University of Indiana, July 1991. 